\title{
POSIBILIDADES Y DIFICULTADES DE UN ANÁLISIS ESPACIAL CONTESTATARIO
}

\author{
José Luis Coraggio \\ El Colegio de México
}

\section{INTRODUCCIÓN}

El PRopósıto de este trabajo está limitado a proveer una base preliminar de discusión sobre algunos de los problemas a los que se enfrentan quienes intentan plantear la cuestión de la organización espacial de una sociedad desde una perspectiva que rompa con los esquemas dominantes caracterizados por un fuerte contenido apologético del sistema social capitalista.

Quienes se abocan a tal tarea encuentran que no se parte de "cero". Contribuciones tan variadas como las de la economía espacial de vertiente neoclásica, la llamada teoría de los polos de desarrollo, la sociología urbana, la denominada geografía teórica, la geopolítica, y' otros cuerpos doctrinario-teóricos representan con variado éxito en este campo específico, la ideología dominante en los sistemas capitalistas. Y no sólo tienen ya una larga historia de investigación - con la producción de conceptos e instrumentos de análisis de creciente "sofisticación"— sino que están en una etapa de reformulación de algunas de las cuestiones críticas que se les plantea desde la realidad contemporánea. Dicha reformulación consiste en adaptar las preguntas a los términos propios de su problemática, con lo que se da así la impresión de tener posibilidades científicas por una mera adaptación o ampliación de sus esquemas, de manera usual mediante la introducción de nuevas variables en los modelos preexistentes. ${ }^{1}$

Tal como ocurre en otros campos, surge la cuestión de qué vía seguir en el camino de superación de las teorías existentes: a) puede intentarse dejar directamente de lado el sistema de teorías, métodos y técnicas, por entender que sus respuestas son puramente "ideológicas" o, más claramente "contrarrevolucionarias". 2 En tal caso, una foma de proseguir

1 Un ejemplo de este esfuerzo por incorporar "lo político" e incluso hasta "los valores morales" bajo la forma de nuevas ecuaciones y variables a un modelo de equilibrio general puede encontrarse en Walter Isard, et. al., General Theory: Social, Political, Economic and Regional, MIT Press, 1972.

2 Para una definición de "teoría contrarrevolucionaria", ver: David Harvey, Social Justice and The City, Arnorld, 1973, Cap. 4. 
sería, por ejemplo, organizar nuevas respuestas a las viejas preguntas a partir de un proceso de deducción o especificación desde teorías generales contestatarias del sistema social; $b$ ) puede incluso dejarse de lado no sólo las respuestas de dichas teorías sino también sus preguntas, en la presunción de que se trata de pseudoproblemas, con lo cual la pertinencia del campo mismo está en duda (esto implicaría, por ejemplo, afirmar que "lo espacial", o "lo regional", o "lo urbano", etc., no tienen especificidad como campos de investigación científica) ; c) con la ayuda de una tópica filosófica o teórica no apologética, embarcarse en el análisis crítico de las contribuciones existentes, al mismo tiempo que se emprenden nuevas investigaciones empíricas, en la situación (nada confortable) de tener que usar algunos de los mismos conceptos y técnicas que están bajo examen crítico (por ejemplo: seguir con la utilización provisoria del concepto de ciudad en su sentido ecológico-demográfico así como las técnicas utilizadas de manera usual para su delimitación). En este caso, un resultado posible es que las cuestiones de partida sean efectivamente rechazadas como no significativas (en cuyo caso queda en tela de juicio la especificidad del campo mismo), o que del proceso crítico surja una nueva problemática que (aun cuando pueda estar referida a idénticos "objetos reales") defina de forma esencialmente distinta el objeto científico a investigar.

Aun cuando las dos primeras alternativas por lógica son posibles y abiertas a la elección personal de distintos investigadores, se considera que el tercer camino es el que ha probado históricamente su eficacia. En tal sentido se parte de una tópica filosófica para repreguntarnos sobre el sentido del "análisis espacial".

\section{Algunos elementos ontológicos ${ }^{3}$}

La cuestión general de la caracterización del espacio en relación a los procesos sociales puede ser planteada con mayor claridad con base en un análisis diferencial de dicha relación, en comparación con la que se da respecto a procesos de naturaleza diferente, así como respecto a la geometría. Para bosquejar tal análisis, se utilizan con profusión materiales extraídos del trabajo de Hartmann. ${ }^{4}$

Adoptamos aquí la posición ontológica, sustentada por los desarrollos :científicos y técnicos, de que la realidad está ordenada en por lo menos culatro estratos, a saber:

3 La relevancia de las cuestiones ontológicas fue traída a mi atención por Raúl Sciarreta, maestro al cual estoy profundamente agradecido por sus estimulantes discusiones sobre la cuestión del espacio.

4 Nicolai Hartmann: Ontología, Vol. 3 (1959), Vol. 4 (1960), México, Fondo de Cultura Económica. Cuando cite dicho texto, en cada caso, el volumen y página correspondientes se indica a continuación. 
a) Vida histórica, colectiva;

b) Vida psíquica;

c) Vida orgánica;

d) Materia inerte.

Esto en el entendimiento de que tal ordenamiento (junto con la concatenación de estratos a través de las categorías que los relacionan) no niega la unidad del mundo real. Por el contrario, esta es precisamente la forma de tal unidad (a diferencia de lo que sería una unidad basada. en la homogeneidad, por ejemplo). Negar alguno de estos estratos, intentando reducirlos a otros, da lugar a reducionismos tales como el biologismo o el ficisismo, por ejemplo, que ignoran la diferente legalidad de estas esferas del ser, así como la distinción entre sus categorías. El desa-rrollo de las ciencias sustenta esta estratificación en el proceso de delimitar sus diferentes objetos de conocimiento. Por otra parte, no se está diciendo que estos amplios estratos sean internamente homogéneos. Diferentes disciplinas pertenecientes a cada gran continente del conocimiento testifican - con sus objetos crecientemente diferenciados- sobre esta gradación interna.

La gradación entre estratos está dada por la irreversibilidad que muestra el análisis categorial al encontrar categorías inferiores como elementos de la estructura de estratos superiores (retorno) aunque nunca encon. trando categorías superiores (es decir, propias de los estratos superiores, en los cuales constituyen el novum) en los estratos inferiores. Por ejemplo: “... (el análisis categorial) se encuentra en las categorías de lo orgánico con las de lo material, pero no en éstas con las de lo orgánico (asimilación, autorregulación, regeneración, reproducción)" [Vol. 3, p. 537]. Si esto no fuere así, “...podría existir la vida orgánica también libremente por sí sin la naturaleza inanimada; la conciencia podría existir flotando libremente sin sostenes orgánicos, el mundo del espíritu sin vida psíquica. El mundo real estaría escindido, desgarrado. Pero por otra parte, si el retorno fuera reversible, no podría haber ser material sin ser orgánico, ni ser orgánico que no tuviese alma y conciencia, ni ser. psíquico que no tuviese espíritu. El mundo tendría demasiada unidad, los estratos coincidirían todos en un solo estrato." [Vol. 3, p. 538]. Pero esto no implica que las categorías de cada estrato superior deban contener todas las de los estratos inferiores.

En otros términos: estos estratos no pueden ser pensados como neutralmente supuestos, como independientes. Hay una dependencia categorial, consistente en que los estratos superiores están sustentados por los inferiores, en el sentida de que al menos algunas de las categorías de un estrato inferior son también categorías de los superiores (aunque sobreconformados en la nueva estructura categorial). De hecho, las categorías de los estratos inferiores pueden ser limitadas al nivel de momentos sub. 
ordinados en un estrato superior..$^{5}$ En las formaciones superiores (ver más adelante), constituyen condiciones, en el sentido de que dichas formaciones no podrían existir sin que se den tales categorías inferiores.

Por otra parte, sería inaceptable pretender una reducción de las formas superiores del ser a las inferiores: “... en la estratificación coexiste el condicionamiento elemental desde abajo con la no debilitada autarquía de los estratos superiores frente a los inferiores..." [Vol. 3, p. 562].

Para clarificar qué se entiende por "condición" y "condicionamiento" puede ayudar tener in mente la que Hartman denomina "ley de la materia" (una de las cuatro leyes de la dependencia): "donde quiera que en la estratificación existe retorno y sobreconformación es la categoría inferior sólo 'materia' para la superior". Sin embargo, "La categoría superior no puede conformar con la materia de la inferior todo lo que quiera, sino sólo lo que es posible en tal materia. No puede transformar los elementos inferiores (pues éstos son más fuertes que ella), sino sólo sobreconformarlos. Más allá de semejante función restrictiva no alcanza el poder determinante de la 'materia'" [Vol. 3, p. 566].

Esta concepción (que no tiene nada en común con una reducción a la materia inerte), aparece con toda claridad en el análisis categorial que Marx aplica a la mercancía:

"Los valores de uso -chaqueta, lienzo, etc., en suma, los cuerpos de las mercancías- son combinaciones de dos elementos: material natural y trabajo. Si se hace abstracción, en su totalidad, de los diversos trabajos útiles incorporados a la chaqueta, al lienzo, etc., quedará siempre un sustrato material, cuya existencia se debe a la naturaleza y no al concurso humano. En su producción, el hombre sólo puede proceder como la naturaleza misma, vale decir, cambiando, simplemente, la forma de los materiales. Y es más: incluso en este trabajo de transformación se ve constantemente apoyado por fuerzas naturales." ?

Pero no sólo la forma natural de la materia ha sido cambiada en el proceso de trabajo. Las mercancías son formaciones superiores con un carácter estratificado:

"Las mercancías vienen al mundo revistiendo la forma de valores de uso

o cuerpos de mercancías: hierro, lienzo, trigo, etc. Es ésta su prosaica

5 Ibid., Vol. 3, Cap. 55.

- Esta relación de "autonomía relativa" (o dependencia y autarquía al mismo tiempo), en tanto fuera aplicable al interior de cada estrato podría ayudar a captar el sentido de la expresión "determinación en última instancia" por la base económica respecto a las instancias político-jurídica e ideológica de las estructuras sociales (o sea la base económica como condición de existencia de los demás procesos sociales, aunque su determinismo esté sobreconformado y sobreconstruído en el todo social).

7 C. Marx, El capital, México, Siglo XXI Editores, 1976, Tomo I, Vol. 1, p. 53 . 
forma natural. Sin embargo, sólo son mercancías debido a su dualidad, a que son objetos de uso y, simultáneamente, portadoras de valor. Sólo se presentan como mercancías, por ende, o sólo poseen la forma de mercancías, en la medida en que tienen una forma doble: la forma natural y la forma de valor." "En contradicción directa con la objetividad sensorialmente grosera del cuerpo de las mercancías, ni un sólo átomo de sustancia natural forma parte de la objetividad en cuanto valores. De ahí que por más que se dé vuelta y se manipule una mercancía cualquiera, resultará inasequible en cuanto cosa que es valor. Si recordamos, empero, que las mercancías sólo poseen objetividad como valores en la medida en que son expresiones de la misma unidad social, del trabajo humano; que su objetividad en cuanto valores, por tanto, es de naturaleza puramente social, se comprenderá de suyo, asimismo, que dicha objetividad como valores sólo puede ponerse de manifiesto en la relación social entre diversas mercancías." 8

Al llegar a este punto de la exposición, puede ser conveniente advertir acerca de la posibilidad de confundir los estratos de la realidad con formaciones complejas reales, las cuales, “... en cuanto tales no son de un estrato, de suerte que tampoco son representantes puras de un estrato del ser. Ahí está, por ejemplo... el 'hombre'; pero el hombre mismo es un ente estratificado, es un ente orgánico, psíquico y espiritual, y ni siquiera falta el estrato inferior, pues en definitiva el hombre es 'también' un ente material” (Vol. 3, p. 214). "...el ser psíquico no contiene en su seno los procesos orgánicos, pero sí los contiene en su seno el 'hombre'; pues el hombre es él mismo un ente estratificado, siendo además organismo y por consiguiente también una formación corpóreomaterial. Por tanto tiene en sí como momentos constitutivos todas las categorías inferiores. Está sujeto a la gravedad, a la presión, a la transformación de la energía tanto como al hambre, a la mortalidad y a la generación" [Vol. 3, p. 540].

De manera análoga podríamos pensar las formaciones socioeconómicas. En los términos de Hartmann: "El espíritu objetivo no contiene, sin duda, actos psíquicos propiamente tales, no es un proceso orgánico ni una formación física; pero sí es todo ello junto la colectividad, así como la historia de ésta, en las cuales y solamente en las cuales existe aquél. Un pueblo tiene su espacio vital, su proceso de vida orgánica a la manera de la vida de las especies animales, con autoproducción y sucesión de generaciones; tiene también su especial índole psíquica y únicamente sobre todo esto se eleva su vida espiritual. En un ente tan estratificado como el individuo humano. Y su historia es un proceso tan estratificado como la vida humana individual" [Vol. 3, p. 540].

En consecuencia, aun cuando los estratos superiores (o algunas de sus instancias internas) pudieran ser considerados como aislados de sus fundamentos ontológicos, a fin de realizar un análisis profundo de su

8 Ibid., Tomo I, Vol. 1, p. 58. 
estructura categorial (y por supuesto, para guiar nuestras acciones), debemos recordar que efectivamente nunca se dan de manera aislada. En caso contrario, otro "ismo" (estructuralismo) obstaculizará nuestra comprensión de los procesos reales, y los métodos apropiados de investigación tenderán a ser reemplazados por la especulación. ${ }^{9}$

\section{El ESPACIO COMO CATEgoRíA}

Dentro del marco de las notas anteriores, se trata de plantear ahora la cuestión del espacio como categoría y sus variaciones ente los distintos estratos.

El momento categorial fundamental del espacio es (como en el caso del tiempo) la dimensionalidad. El espacio es la condición categorial de lo extenso, pero no es "extenso" en sí mismo. Negar esto conduce a una sustancialización del espacio. El espacio no existe. Como todas las categorías, su modo de ser es de otra naturaleza, o sea la esencia de las categorías es inherente,... el no tener más ser que su ser principios para lo concreto" [Vol. 4, p. 53]. "El espacio (y el tiempo) no tienen, en absoluto, existencia real alguna fuera y al lado de las cosas y los procesos reales de que son las dimensiones reales" (Vol. 4, p. 52].

Una diferencia significativa entre el tiempo y el espacio como categorías tiene que ver con su variación a lo largo de la estratificación del ser. El tiempo es (con variaciones) una categoría para todos los estratos de la esfera real, pero no lo es de la esfera ideal (los objetos ideales son intemporales), mientras que el espacio es tanto una categoría real como ideal (aunque en la esfera real esté limitado a los estratos naturales). En otras palabras, ambos son categorías sólo para el dominio natural, donde “... son todas las formaciones y procesos de índole a la vez espacial y temporal, o sea, que suponen las tres dimensiones del espacio y del tiempo" [Vol. 4, p. 47].

Hartmann distingue tres modos de ser del espacio: el espacio ideal, el espacio real y el espacio intuitivo. Nos restringiremos a una breve consideración sobre los dos primeros.

\section{EL ESPACIO IDEAL}

Algunos de los momentos categoriales del espacio ideal presenta. dos por Hartmann [Vol. 4, Cap. 5], son los siguientes:

a) Es un puro sistema de dimensiones de magnitud extensiva;

b) Es homogéneo (toda diferenciación de lugares del espacio es relativa a algo que ocupa un lugar en el espacio);

9 Para un intento productivo de superar estas dificultades, ver los trabajos de la escuela institucionalista francesa. Por ejemplo: George Lapassade y René Lourau Clefs pour la sociologie, París, Editions Seghers, 1971. 
c) Es continuo (divisible hasta el infinito);

d) Es ilimitado (aunque haya límites "en" el espacio);

e) No provee de suyo ningún patrón de medida (aunque determina "la especie" de dicho patrón);

f) Sus dimensiones son homogéneas (pueden ser intercambiadas entre sí. Es por tanto, isométrico).

La geometría constituiría la ciencia del espacio ideal. Si bien la concepción euclideana ha dominado más de dos mil años de la historia del pensamiento, recientemente han surgido nuevos sistemas geométricos (la geometría hiperbólica de Lobachevski, o la elíptica de Riemann) que han probado ser internamente consistentes como la euclideana. Por lo tanto, no habría criterios formales para optar entre una $\mathrm{u}$ otra. La cuestión de qué geometría es la adecuada para su aplicación a la resolución de problemas de medición y análisis espacial de orden real debe entonces ser resuelta en función de una concepción científica del espacio real. ${ }^{10}$

\section{El espacio real}

"El espacio real es el espacio en que existen las cosas reales y las relaciones entre estas cosas, en que se desenvuelven los sucesos reales físicos, en que transcurre también la vida humana, en la medida en que su curso es el natural de las cosas y está sujeto a condiciones naturales, tanto la vida individual cuanto la colectiva e histórica. El espacio real es exactamente tanto espacio cósmico como espacio vital, campo en que entran en juego cuerpos y fuerzas cósmicas y campo en que entra en juego el hacer y el deshacer del hombre. Es el espacio de lo existente, la forma y condición categorial del mundo exterior”. [Vol. 4, p. 93.]

En lo que respecta a la estratificación del mundo real, la categoría real de la espacialidad abarca sólo los dos estratos inferiores: "...por encima de lo orgánico ya no ocurre separación ni relación extensivoespacial alguna" [Vol. 4, p. 94].$^{11}$

Los momentos categoriales del espacio ideal enumerados antes se aplican también al espacio real. Sin embargo, hay algunas diferencias significativas, de manera principal la singularidad y tridimensionalidad del espacio real. Sobre la base de este análisis categorial, Hartmann rechaza versiones modernas del espacio como una función de fuerzas, del mismo modo que han sido rechazadas viejas concepciones del espacio como

10 Sobre este tema, puede consultarse: Harvey, David: Explanation in Geography, Londres, Arnold, 1969, Capítulo 14.

11 Para mayor claridad: esto no resta significación a la espacialidad de formaciones tales como individuos o mercancías o en general objetos sociales, así como tampoco a las formas espaciales identificables referidas a ellos. Sin embargo, esta espacialidad no les corresponde en tanto objetos sociales, sino en tanto tienen un sustrato natural. 
un accidente de la materia. Tal como él lo plantea: “...no son el volumen, la distancia, el movimiento mismos, el espacio, sino algo 'en' el espacio. Aunque sean, pues, obras de la fuerza ..." "... la relación de la fuerza con el espacio es la misma que la de la materia con él" [Vol. 4, p. 101]. En todo caso, lo que suele denominarse como espacio de fuerzas o como espacio vital, "no son, patentemente, especies del espacio real, sino dominios dentro del mismo espacio, deslindados dentro de éste por el alcance de ciertas relaciones, las cuales son, por su parte, espaciales y pueden entrecortarse espacialmente... Es un contrasentido atribuir al espacio lo que conviene a las solas formaciones que tienen su dimensión en él" [Vol. 4, p. 66].

Lo que se ha expuesto no debe ser interpretado en el sentido de que el espacio deba ser considerado como un receptáculo para las cosas, sino como constitutivo de las cosas.

\section{LA RELACIÓN ENTRE EL ESPACIO GEOMÉTRICO Y EL ESPACIO REAL}

Comparado con la multiplicidad de espacios geométricos, el espacio real es sólo un caso especial. Por lo tanto, sólo una geometría debería ser propia de las relaciones espaciales reales, aunque para Hartmann la cuestión sobre cuál de las geometrías conocidas es la apropiada estaría aún pendiente. En cualquier caso, la relación entre el espacio real y el correspondiente espacio geométrico “....consiste en la validez de las leyes geométricas para las cosas y las relaciones entre ellas que se extienden en el espacio real” [Vol. 4, p. 83]. Así: “... los cálculos que sobre la base de la geometría pura hacemos con los objetos reales se confirman en la experiencia; la ciencia exacta de la naturaleza y la técnica descansan en ellos..." [Vol. 4, p. 84]

Por supuesto que podríamos limitarnos a considerar las extensiones, formas, posiciones, distancias y direcciones sobre la superficie de la tierra, juntamente con la necesarias relaciones involucradas. Pero en ningún caso podrían nuestros cálculos violar la geometría pura del espacio real, “en" el cual están la tierra y los objetos corpóreos bajo estudio. Esta utilidad instrumental no puede ser ignorada, pero - y aquí está la diferencia fundamental con ciertas corrientes "espacialistas"- de ninguna manera podría la geometría constituir la lógica y aún menos la teoría de los procesos reales, aun cuando éstos sean espaciales. ${ }^{12}$

12 Desde un punto de vista diferente, véase William Bunge, Theoretical Geography, Lund Studies, 1966, y para una breve discusión de algunas de las proposiciones allí expuestas: José L. Coraggio, "Consideraciones teórico-metodológicas sobre las formas sociales de organización del espacio y sus tendencias en América Latina", Revista Interamericana de Planificación, Vol. VIII, Núm. 32, diciembre de 1974. 


\section{LA ESPACIALIDAD Y LOS PROCESOS DE ORDEN SUPERIOR}

Cuando nos referimos al espacio real ilustramos la mayoría de las proposiciones planteadas con ejemplos relacionados a cuerpos materiales. Dentro del segundo estrato real, el de la vida orgánica, el espacio es aún un momento esencial. El organismo tiene tanto una forma espacial exterior como una configuración espacial interna. Sin embargo, el significado de esta última sólo puede ser aprehendido desde el punto de vista de procesos tales como los de desarrollo, autorregulación y autorreproducción, todos los cuales constituyen una novum en este estrato.

En el estrato superior siguiente estamos ya en lo propiamente inespacial: "La vida psíquica ya no tiene forma espacial; sus contenidos no son formaciones espaciales" [Vol. 4, p. 125]. Sin embargo, la conciencia sólo puede existir si está sustentada por un organismo, y con ello: “...no está sólo la conciencia ligada indirectamente al espacio, sino que también su saber del mundo circundante es un saber orientado espacialmente" [Vol. 4, p. 125]. "Esta espacialidad indirecta de la conciencia es de una especie muy distinta de la del organismo y, sobre todo, de la de las cosas materiales, pero sigue siendo siempre una especie de espacialidad" [Vol. 4, p. 125]. “... en la misma relación descansa, además, la bien conocida espacialidad geográfica de las culturas, de los sucesos de la historia y de la vida colectiva del espíritu... sustentados por una pluralidad de individuos vivientes" [Vol. 4, p. 126].

\section{Tratamientos alternativos del espacio en relación a los PROCESOS SOCIALES}

Las notas precedentes han permitido plantear de manera esquemática la tópica filosófica desde la cual nos proponemos orientar nuestra revisión crítica de las concepciones de lo espacial en las ciencias sociales. Sobre esta base, podemos visualizar al menos tres caminos alternativos principales para encarar la cuestión del espacio en relación a los procesos sociales. El primero consistiría en la reducción al espacio físico. Tal vía indicaría que la investigación de aquellas formas espaciales de materia sobre la superficie terrestre que resultan de procesos sociales debería ser emprendida a través de la extrapolación de categorías propias del espacio físico. Esto puede ser hecho explícita o implícitamente como en el caso de algunas aplicaciones de modelos físicos gravitacionales al estudio de la distribución de la población o del desplazamiento de población o mercancías. En esta perspectiva, qué tipo de teoría física (y la correspondiente geometría) proveería el más adecuado paradigma para los estudios sociales es una cuestión a ser resuelta. ${ }^{13}$

13 Véase por ejemplo: los recientes trabajos de Walter Isard publicados en 
A nuestro juicio, pueden "importarse" de manera legítima modelos cuantitativos de otras ciencias a fin de explorar nuevos campos de información, en el entendimiento de que son utilizados en su forma transitoria y meramente limitados al nivel operativo de la investigación de fenómenos. Lo que no podríamos aceptar es la extrapolación de categorías de un estrato al otro de la realidad, tal como se hace cada vez que la legalidad de los procesos sociales es asimilada a la de los físicos.

Un segundo camino sería el derivado de centrar la atención en un enfoque diferente del análisis categorial del espacio. De acuerdo a Hartmann, la posición neokantiana al respecto consiste en entender al espacio como la forma de "estar junto", y al tiempo como la forma de "estar separado" de las formaciones reales. De esta manera, se lleva la simultaneidad, que es sólo un modo determinado de la temporalidad, a la posición de criterio crucial para establecer la diferencia y por tanto la verdadera naturaleza del tiempo y del espacio. Así, en la teoría de la relatividad, el espacio es visualizado como una relación de coexistencia en la situación de los̀ cuerpos materiales.

Si consideramos entonces al espacio como un sistema de relaciones simultáneas, entonces la variación de esta categoría al entrar al estrato histórico de la realidad, estaría dada por el hecho de que, por ejemplo, las relaciones sociales son de naturaleza distinta a las del estrato inorgánico. En cierto modo, esto es coherente con los enfoques estructurales del estudio de la sociedad. Así, podríamos hablar de un "espacio social", oponiéndolo al espacio físico, lo cual quiere decir que los sistemas sociales y los físicos son de naturaleza diferente. Pero si de esto se trata, no parece necesario hablar en términos de "espacios". Podríamos referirnos de manera directa a sistemas o complejos, como formaciones específicas con diferente estratificación y proceder a investigar cómo diferentes estructuras están simultáneamente presentes en la entretejida malla de dichas funciones. No hay duda de la relevancia de tal curso de investigación para aprehender los procesos históricos, pero ésta no es nuestra materia específica de estudio (el análisis espacial referido a fomaciones sociales).$^{14}$

Papers and Proceedings, Regional Science Association, y en particular sus proposiciones en "Why is the General Relativity Theory Relevant to Regional and Other Social Sciences", en R. Funk, Recent Developments in Regional Science, Londres, 1972.

14 Otra forma en que se podría dar el "paso" de los espacios físicos a los "sociales" con la conservación de la forma de "sistema de relaciones" podría ilustrarse así: un espacio está definido por un sistema de relaciones espaciales entre elementos individualizados por su posición relativa en un sistema de coordenadas (por ejemplo: latitud y longitud). Cada individuo representado por un par de coordenadas $(x, y)$ y las distancias (relaciones espaciales) entre individuos definidas según las reglas de la trigonometría elemental $d^{2}=\left(x_{1}-x_{2}\right)^{2}+\left(y_{1}-y_{2}\right)^{2}$. $\mathrm{Si}$ ahora pasamos a individualizar los elementos a través de un vector de atributos no espaciales $(z, q, w, \ldots)$ que están referidos a un sistema de coordenadas corres- 
Un tercer camino sería continuar el análisis categorial siguiendo las variaciones de la espacialidad a través del estrato histórico, sobre la idea de la espacialidad indirecta como la presenta Hartmann. Tal como lo vemos hoy, esta es la mejor manera de proceder (dados nuestros objetivos iniciales de realizar una revisión crítica de las teorías y métodos existentes en el campo) porque permitirá analizar de manera crítica algunos problemas específicos presentados por lo común bajo el título de "análisis espacial", dentro de un marco filosófico y teórico adecuado. Por otra parte, este enfoque no excluye al segundo, en tanto las formaciones sociales constituyen el objeto general de nuestra investigación.

\section{LA ESPACIALIDAD INDIRECTA DE LOS PROCESOS SOCIALES}

Un buen comienzo para investigar la relación (espacialidad indirecta) entre procesos sociales y formas espaciales es el que provee la relación básica del hombre con la naturaleza a través del proceso de producción. $\mathrm{Si}$ nos restringimos a considerar las determinaciones más simples del metabolismo económico en una sociedad con un alto grado de división social del trabajo, podemos visualizarlo como una serie de cadenas de operaciones de producción, circulación material y consumo de productos. ${ }^{15}$

Tan pronto como el nivel de organización social del metabolismo económico resulta en su repetición más o menos regular, se producen cristalizaciones espaciales tales como: a) localización de medios de trabajo requeridos para las operaciones de producción (edificios, maquinaria, mejoras agrícolas, etc.); b) localización de medios de trabajo para las operaciones de transporte (centros de trasbordo, todo tipo de ductos, vías férreas, caminos, etc.); c) localización de medios de trabajo para las operaciones de almacenamiento (depósitos en general, silos, tanques, comercios, etc.) y; d) localización de medios de vida (vivienda, en su sentido más amplio). Asimismo, en la medida que los flujos de productos entre los lugares de los diferentes centros de producción y consumo se vuelven repetitivos, podemos hablar también de una configuración espacial cristalizada de la cirçlación material requerida por el proceso metabólico.

Sin este metabolismo ninguna sociedad podría subsistir. A su vez,

pondientes a cada dimensión o atributo (ingreso, edad, años de estudio, etc.), con sólo extrapolar la definición de distancia en un espacio de $n$ dimensiones estaríamos "habilitados" para hablar de un "espacio social" (en tanto los atributos sean de ese orden) y de "relaciones o distancias sociales". Véase, por ejemplo, las aplicaciones del análisis factorial. Un intento de fundamentar explícitamente este "paso", puede verse en Francois Perroux, "Economic Space: Theory and Applications", Quarterly Journal of Economics, Vol. 64, febrero de 1950.

15 Para un desarrollo más detallado de estas operaciones, véase José L. Coraggio, op. cit. 
este sistema de operaciones de transformación no puede funcionar sin el sustrato material que proveen los medios de producción y la fuerza de trabajo, todos ellos de naturaleza espacial. Tenemos aquí un claro elemento de espacialidad indirecta, sobre cuya base se abren numerosas cuestiones, como por ejemplo: ¿cómo está regulado este complicado metabolismo? ¿cómo se dio su génesis y posterior desarrollo? ¿qué tipos de valores de uso produce? ¿cómo están distribuidas las fuerzas productivas entre las diversas ramas de actividad? $O$ de manera más espe. cífica: ¿cómo se regula la distribución geográfica de los medios de producción y de los flujos de productos? ¿cómo se regula la distribución y redistribución de la fuerza de trabajo? $\mathrm{O}$, aún más específica: ¿hay formas espaciales estables en esta organización que aparezcan en forma recurrente en diferentes lugares del mismo sistema y en otros sistemas también? Si pueden identificarse formas espaciales estables y repetitivas ¿qué tipo de determinismo las produce? ¿es un determinismo natural, es decir que dichas formas surgen de procesos naturales y son por lo tanto tan universales como lo son las leyes de la naturaleza? o, ¿es un determinismo social, el cual significa que estas formas son un puro resultado de procesos sociales y son por lo tanto de carácter histórico, pero además "reflejando" procesos y relaciones sociales (representantes puros del estrato histórico)? En otros términos (y otro enfoque) : ¿qué tipo de funcionalidad tienen diversas formas espaciales respecto a la sociedad concreta en la que se dan? Si las estructuras sociales se modifican a partir de determinada situación histórica ¿deberán cambiar también las configuraciones espaciales preexistentes? ¿pueden formas espaciales similares "sustentar" estructuras sociales diferentes? $\mathrm{O}$, de otro modo: $i$ son instrumentales para el cambio de las relaciones sociales ciertos cambios en las configuraciones espaciales de la producción, de la población, etc.? Por último, nuestra inquietud cotidiana: ¿cómo deberá regularse la investigación a fin de contribuir a contestar o formular apropiadamente estas y otras preguntas del mismo tenor?

No tenemos ninguna fórmula lista para contestar esta última pregunta. En cambio, nos limitaremos a plantear e ilustrar algunos problemas relacionados a esta cuestión que podrían proveer cierta base para organizar una discusión que está en sus comienżos.

\section{ANálisis ESPACial, DETERMinismo Estratificado Y NECESidAd DE UNA TÓPICA}

Cuando proponemos comenzar la investigación donde el hombre y la naturaleza se relacionan, estamos basándonos en un hecho simple pero crucial: en términos generales, encontramos que las mercancías, además de ser objetos sociales, tienen en general un soporte material provisto por su forma de materia inerte $u$ orgánica de ser. Como conse- 
cuencia, son también espaciales, y los momentos de la espacialidad (extensión, posición, dirección de movimiento, etc.) se les aplica. Esto es así no sólo para los bienes de consumo personal, sino también para las materias primas y medios de trabajo utilizados de manera directa en cada proceso de trabajo, así como para el conjunto del aparato que provee las condiciones generales para la producción $\mathrm{y}$, por último, para la misma fuerza de trabajo.

El caso de los servicios parece plantear ciertas dificultades a esta caracterización general, en tanto son efectos útiles sin un cuerpo físico. Sin embargo, la espacialidad indirecta aparece de todas formas, no sólo debido a la necesaria posición relativa de productores y consumidores de servicios personales al momento del consumo, sino por la necesidad de medios de producción que, a su vez, son espaciales (por supuesto, lo que se afirma acerca de las mercancías se aplica también a los bienes producidos bajo diferentes formas sociales).

Al producir estos bienes, el hombre sobreconforma (pero, de manera necesaria también adapta sus operaciones a) el determinismo general y específico de la materia. Lo mismo ocurre con su propia naturaleza orgánica, que condiciona (considérese lo antes expuesto) sus actos sociales. Como ejemplo ilustrativo, la jornada laboral tiene un límite temporal máximo:

"No es prolongable más allá de determinada linde. Ese límite máximo está determinado de dos maneras. De una parte, por la barrera física de la fuerza de trabajo. Durante el día natural de 24 horas un hombre sólo puede gastar una cantidad determinada de fuerza vital. Así, de manera análoga, un caballo sólo puede trabajar, promedialmente, 8 horas diarias. Durante una parte del día la fuerza debe reposar, dormir, mientras que durante otra parte del día el hombre tiene que satisfacer otras necesidades físicas, alimentarse, asearse, vestirse, etc." 16

Pero en tanto el trabajo es una actividad social, la sobreconformación de las categorías inferiores por las superiores aparece inmediatamente:

"Aparte ese límite puramente físico, la prolongación de la jornada laboral tropieza con barreras morales. El hombre necesita tiempo para la satisfacción de necesidades espirituales y sociales, cuya amplitud y número dependen del nivel alcanzado en general por la civilización. La variación de la jornada laboral oscila pues dentro de límites físicos y sociales. Unos y otros son, sin embargo, da naturaleza muy elástica y permiten la libertad de movimientos." 17

"En la historia de la producción capitalista la reglamentación de la jornada laboral se presenta como lucha en torno a los límites de dicha

16 C. Marx, op. cit., Tomo I, Vol. 1, p. 278.

17 Ibid., Tomo I, Vol. 1, p. 279. 
jornada, una lucha entre el capitalista colectivo, esto es, la clase de los capitalistas, y el obrero colectivo, o sea la clase obrera." 18

Es claro aquí que el condicionamiento físico sobre la jornada laboral no constituye un determinismo directo y completo sobre la misma.

$\mathrm{Y}$ del mismo modo que en el mundo real el movimiento ocurre en las cuatro dimensiones del espacio y el tiempo, los límites naturales de la jornada laboral también establecen límites en el tiempo y el espacio al viaje cotidiano del trabajador hacia y desde su lugar de trabajo. Cuanto más prolongada sea la jornada laboral, tanto menor será el tiempo disponible para el transporte de los obreros entre lugar de trabajo y de residencia, dado un tiempo mínimo necesario para la reproducción efectiva de su fuerza de trabajo. Si bien es evidente que el tiempo involucrado en el desplazamiento hacia y desde el lugar de trabajo no es contabilizado (y mucho menos pagado) como parte de la jornada laboral, no es correcto considerarlo sólo como parte del tiempo dedicado a la reproducción (tanto más bajo las deplorables condiciones que prevalecen en tantas grandes metrópolis para el desplazamiento de los trabajadores). Dadas ciertas condiciones del transporte, este desplazamiento cotidiano también tiene límites de distancia que, a su vez, ponen límites a la distancia relativa entre lugares de trabajo y de residencia. Ahora bien, aun cuando estos límites tienen un condicionamiento natural, no podríamos decir que el patrón de localizaciones relativas de los lugares de trabajo y de residencia de los trabajadores esté determinado por leyes naturales. No se trata sólo de que la jornada laboral ya tiene una determinación social, sino que la configuración espacial misma de las ciudades está en parte determinada por la intervención del Estado y por las luchas sociales acerca de las condiciones de transporte y de la repoducción de la fuerza de trabajo. ${ }^{19}$

Si desde las regulaciones legales relativas a la localización de industrias, hasta la cuestión de la vivienda, en cada aspecto de los "progra. mas urbanos" se pudiera encontrar esta sobreconformación de categorías naturales por parte de las históricas, ¿cómo podría aceptarse la proposición de que las diferentes posiciones relativas de las residencias de diversos grupos sociales en el campo urbano es un resultado de la ley universal de gravitación y del hecho de que los individuos de diferentes grupos sociales tienen distintas percepciones del espacio? $\mathrm{O}$ consideremos la proposición de que la dirección de los vientos y la posición de las corrientes de agua superficial determinan la localización de las industrias contaminantes. (Sin saberlo, la teoría de la localización indus-

18 Ibidem., Tomo I, Vol. 1, p. 282.

19 Sobre este tema, los trabajos de Manuel Castells son fundamentales. Véase por ejemplo: La cuestión urbana, México, Siglo XXI Editores, 1974, y también: "Urban Contradictions and Collective Consumption in Advanced Capitalism", en L. Lindberg (Comp.), Sources of Change in Advanced Industrial Societies, 1974. 
trial usa un buen término para estas determinaciones: se las denomina "factores". Y eso es lo que son. De ningún modo son "causas", sino condiciones sobreconformadas por los procesos sociales). Tomemos aún otro ejemplo: en el caso de la extracción de minerales localizados, parecería que la determinación de la localización de la actividad extractiva por la localización de los depósitos naturales es completa. Sin embargo, para que las formas naturales de la materia se conviertan en "recursos naturales", es necesario un proceso histórico de desarrollo del metabolismo social, con referencia al cual constituye un recurso. Aún más, el hecho de que no todos los depósitos de minerales conocidos (y el preguntarnos: conocidos ¿por quién? también nos diría algo) estén en explotación, y la misma secuencia histórica de entrada de los diferentes depósitos al mercado mundial, muestran que de ninguna manera los factores naturales "explican" por sí mismos la localización de las actividades. ${ }^{20}$

Por lo tanto, no podemos reducir el determinismo de la configuración espacial de los objetos materiales que soportan los procesos sociales, a un determinismo natural. Pero cabría preguntarse: ¿podríamos reducirlo a un determinismo geométrico? En otras palabras, ¿existen "leyes espaciales" que regulan los fenómenos de diferentes estratos de la realidad, incluido el histórico ${ }^{21}$

Por supuesto, se pueden encontrar ciertos isomorfismos entre la configuración espacial de cuerpos socialmente producidos y consumidos y de los individuos mismos, por un lado, y la correspondiente configuración de algunos fenómenos físicos, por el otro. Incluso se podría encontrar un isomorfismo directo con formas geométricas regulares. Por ejemplo, en el caso del diseño de las unidades de producción agrícola en algunas áreas, así como de la disposición relativa de las viviendas agrícolas respecto al campo agrícola mismo. Se podría encontrar una relación funcional sobre la base de la organización "racional" de los movimientos involucrados en el proceso de trabajo, junto con ciertas limitaciones espaciales puras ( por ejemplo, un sistema de áreas de producción circulares y no superpuestas, no puede cubrir el conjunto de una área agrícola dada, lo que, a su vez, requiere una restricción "externa" adicional, o sea que no haya intersticios). Siendo la geometría la ciencia del espacio ideal, se considera factible encontrar en las leyes geométricas la explicación de estas formas producidas por procesos sociales. Como se

20 Para un análisis de la entrada de los depósitos venezolanos de mineral en el campo de interés de los monopolios, véase Lyzbeth Thismon Mañe, La teoría de los polos de desarrollo y su relación con las politicas de desarrollo regional en Venezuela. El caso de Ciudad Guayana, Centro de Estudios Urbanos y Regionales (CEUR), Programa de Formación de Investigadores, Informe de Tesis, 1975.

21 Por ejemplo, en W. Bunge, Theoretical Geography, Lund, 1966, se habla de leyes como la de la "regla de desplazamiento", que permitiría predecir desplazamientos de rutas camineras, ríos, centros comerciales, geysers, etc., con base en ciertos isomorfismos espaciales entre dichos fenómenos. 
sabe, una explicación de estos procesos sociales y de los aspectos específicos de su espacialidad indirecta no provendrá de la geometría, aun cuando ésta puede proveer recursos formales abstractos para formular una explicación. Esto es así, porque el significado social de las formas geométricas que reconocemos entre los soportes materiales de los procesos sociales no es un significado geométrico. El significado de dichas forrnas está en procesos reales de orden histórico, que no pueden ser aprehendidos por la geometría. ${ }^{20}$

Así, hemos llegado a un punto decisivo: a fin de buscar el significado de las formas espaciales de la materia natural que le han sido estampadas por procesos históricos, es necesario ajustar nuestra investigación a la estructura real de un determinismo múltiple que, en el caso de los productos sociales, está canalizado dentro de un sistema estratificado, cuyo estrato superior es el histórico.

Se requiere entonces partir de una tópica ${ }^{23}$ teórica que perrnita comprender el proceso de producción-reproducción de las formaciones sociales. Esa tópica, tal como ha sido desarrollada por Marx (para partir de un núcleo teórico que ha probado su eficacia histórica), está basada en el análisis categorial del modo de producción capitalista. ${ }^{24}$

$\mathrm{Si}$ adoptamos la versión althusseriana, entenderemos por modo de producción la articulación de las instancias conocidas: la económica, la político-jurídica y la ideológica. A su vez, por formaciones económicosociales entenderemos la combinación de diversos modos de producción (con predominio de uno). Sin embargo, la formación económico social no es la sociedad concreta real (histórica) con la que nos enfrentamos en nuestra investigación, sino que es de orden formal concreto (es decir, se trata de un sistema de conceptos). Por otra parte, en cualquier sociedad particular (una colectividad dada en un determinado momento histórico), se hallan presentes otras gradaciones del estrato histórico, como por ejemplo lo cultural (la lengua, las costumbres, la historia en común, etc.), que escapan a la concepción de la formación económico social.

Por lo tanto, lo que en general constituye una tópica ${ }^{25}$ adecuada para la investigación de fenómenos sociales concretos no es el modo de pro-

22 Lo mismo ocurre con las formas internas de los seres orgánicos, que pueden ser comprendidos solamente desde el punto de vista de los procesos de desarrollo, auto-regulación y reproducción, propios de ese orden (véase Hartmann, op. cit., Vol. 4, Cap. 7).

23 En el sentido de "sistema de lugares" o de ordenamiento de materias.

24 En la obra de Hartmann no se encuentra un desarrollo de las categorías propias del estrato histórico. En la obra científica de Marx es donde puede encontrarse la base para dicho desarrollo, sin duda incompleto al momento de su muerte.

25 Una tópica no debería ser tomada como algo estático, dado y definitivo. Constituye un esquema de ordenamiento para la investigación, pero no contiene los resultados específicos de la investigación en realización. Tales resultados deben ser reinscritos en la tópica, en un proceso continuo de enriquecimiento y rectificación. 
ducción mismo (que es abstracto formal), sino la formación económico social, puesto que no se conoce ninguna sociedad que sea representante pura de un único modo de producción. Además, como se expresó antes, existen otros elementos históricos en una sociedad concreta que no están considerados en el concepto de formación económicosocial tal como se ha desarrollado hasta ahora. ${ }^{26}$

No se intenta profundizar en esta cuestión, que supera los límites de este trabajo, sino que nos concretaremos a hacer algunos comentarios que están relacionados de manera más directa en el tema que nos ocupa. Si se parte de la base de que ya ha sido rechazada la posibilidad de buscar en la geometría, o en los modelos físicos, los criterios reguladores. de nuestra investigación, y que hemos adoptado la tópica de las formaciones económicosociales, aún existe el riesgo de incurrir en vicios de otro tipo cuando encaramos la investigación de la organización espacial de sociedades concretas. Ilustraremos tales dificultades (mediante la presentación esquemática de la estratificación de la realidad hecha antes) como derivadas de la no consideración estricta de la naturaleza estratificada-del determinismo, y de las diversas articulaciones que puede tener en diferentes sociedades concretas.

\section{Algunas posibles dificultades en la investigación de las FORMAS ESPACIALES}

Un caso posible es aquel en que una concepción de la principal corriente de determinismo social que opera en determinados procesos de una sociedad concreta se supone válida para todas las sociedades bajo: el mismo modo dominante de producción. Para ilustrar esta posibilidad, tomemos la siguiente proposición de Castells: “...en las sociedades capitalistas avanzadas, el proceso que estructura el espacio es el referente a la reproducción simple y ampliada de la fuerza de trabajo..." y que: "Las 'unidades urbanas' serían en el proceso de reproducción lo que las empresas son en el proceso de producción... ${ }^{27}$ Por lo tanto, lo que nos propone es estudiar "...le processus social de production d'un système urbain a partir de la logique de la reproduction de la force de. travail ...".28

En otras palabras, Castells plantea la hipótesis de que - dentro de la vasta estructura de categorías que la tópica de la formación económicosocial provee- la atención de los investigadores debería estar cen-

26 $\mathrm{Si}$ se adopta el esquema de definiciones alternativo, consistente en restringir el término "modo de producción" a la instancia económica y se considera como formación económica social la articulación de las tres instancias, etc., esto no modificaría el sentido de las proposiciones que se harán más adelante.

27 M. Castells, op. cit., p. 280.

28 M. Castells y F. Godard, Monopoldville: L'entreprise. l'Etat, l'urbain, París, Mounton, 1974, p. 12. 
trada (aunque por cierto no propone una reducción) sobre los conceptos relacionados a la categoría de reproducción de la fuerza de trabajo. Su propuesta puede ser correcta (y la riqueza de sus recientes contribuciones parece probar lo fértil de tal hipótesis), pero esto no implica que la misma proposición se aplique a cada sociedad capitalista. ${ }^{29}$

Por lo tanto, y hasta que investigaciones adicionales en sociedades capitalistas no avanzadas permitan o induzcan una mayor especificación, podría ser más propio proponer el proceso de producción-reproducción del capital como núcleo categorial para guiar investigaciones sobre la organización espacial (centrada o no en lo urbano) en sociedades capitalistas de América Latina. ${ }^{30}$

Lo anterior se caracteriza como un desplazamiento interno a la tópica de la formación económicosocial. Otro caso, que proponemos caracterizar como interno a la tópica histórica pero externo a la de la formación económicosocial, es el resultante de no advertir que una sociedad concreta es algo más que una trama de procesos económicos, políticos e ideológicos. En términos más concretos: al investigar los procesos de organización espacial (y desarrollo regional desigual) que operan en muchos países de América Latina, ¿se podría ignorar la existencia de diferencias étnicas no sólo entre países sino dentro de cada uno de ellos? ¿no es ésta una "materia" (véase el inciso II) para la sobreconformación por parte de las estructuras sociales, y por tanto un factor condicionante, en algunos casos de gran importancia? Si es así, en los 'estudios sobre tales países o regiones, la tópica de la formación económicosocial debe ser integrada en forma explicita con tales factores étnicos. Al hacerlo, una mejor comprensión general de las complejas articulaciones entre las categorías de estas "gradaciones del ser histórico" podría constituir también un resultado importante.

Un tercer caso, que proponemos caracterizar como desplazamiento externo a la tópica histórica, es el resultante de no advertir que, aun cuando en las formas económicosociales el estrato histórico es el superior y decisivo, aún son formaciones estratificadas. En otras palabras, aunque sobreconformado, el determinismo natural está presente con diferentes grados de importancia en las sociedades concretas. Tales variaciones de grado son debidas sólo a las formaciones naturales específicas de los distintos territorios (¿deberíamos llamarlo "determinismo geográ'fico"?). Dependen también del desarrollo alcanzado por las estructuras sobreconformantes. Para evitar interpetaciones erróneas de lo dicho,

29 En la introducción del libro Estructura de clases y política urbana en Amé. rica Latina, Manuel Castells (Comp.), Ediciones SIAP, 1974, se lee: “... intentamos aplicar a la realidad latinoamericana la caracterización que se ha revelado plenamente operativa en numerosos análisis sobre las sociedades capitalistas avanzadas en Europa, de los procesos urbanos en tanto que procesos estructuralmente definidos por su inserción en la reproducción de la fuerza de trabajo...".

30 Véase José L. Coraggio, op. cit. 
ilustremos este caso con unos pocos ejemplos. Tomemos un fenómeno hipotético: el principal cultivo de un país se pierde debido a condiciones meteorológicas adversas. Los efectos de este evento pueden ser muy diferentes. En una sociedad socialista planificada, con un desarrollo económico complejo, y donde el bienestar de la población es un verdadero principio regulador, podría requerir un ajuste temporal de la asignación de recursos y de las pautas de consumo. En una sociedad capitalista avanzada podría tener los mismos efectos y además un efecto de desempleo temporal y de difíciles condiciones de reproducción para un sector de la población. En un país capitalista atrasado podría tener no sólo efectos de desempleo generalizado, sino también de hambres y muertes masivas. También podríamos ilustrar este caso con el proceso natural de reproducción de la población humana, o proceso demográfico io no es cierto que el determinismo demográfico juega un rol muy distinto en el proceso de organización espacial de diferentes sociedades concretas? Veamos entonces que, bajo distintas formas, el determinismo natural está aún presente en las formaciones económicosociales y que debería ser incluido (con cabal consideración de su sobreconformación) en la investigación del desarrollo regional. Su relevancia para dar cuenta de fenómenos sociales variará con el grado de sobreconformación al que esté sujeto, el que a su vez depende del desarrollo al canzado por las estructuras sociales. ${ }^{31}$

En resumen, lo que se trata de afirmar es que, a fin de evitar el error de extrapolar categorías físicas o aún geométricas (con la correspondiente legalidad) al estrato de los procesos históricos, pero también de evitar los errores de un "análisis espacial" en el vacío, que a lo sumo podría dar nueva forma a los reportes de las construcciones ideológicas dominantes, con la introducción sin base científica de términos tales como el de "espacio social", o el de "dominación espacial", etc., debemos recurrir a la tópica teórica existente más pertinente, que es la de la formación económicosocial. Pero también decimos que, al hacerlo, y a fin de evitar caer en la especulación, debemos referir nuestras investigaciones a sociedades y procesos concretos, ${ }^{32}$ lo que requiere asimismo la

31 Es interesante observar cómo la fuerza del determinismo natural en países c regiones atrasados, con estructuras sociales "débiles", reaparece cuando las sociedades avanzadas pierden "control" de los procesos naturales. Aparece bajo la forma del avance de la erosión del suelo, de la contaminación creciente de la atmósfera y del agua, o del número creciente de casos de deformaciones de nacimiento. Por supuesto, este efecto de determinación natural está en gran medida "disparado" desde las nismas estructuras sociales de un sistema social antagónico.

32 Por desgracia, hay demasiados casos de investigaciones en que se trata de fundamentar proposiciones acerca de la organización espacial en países capitalistas a partir de la mera consideración de la teoría del modo de producción o de manera aún más rudimentaria, se procura "armar" una teoría alternativa a la dominante sobre la base de extraer de los textos de Marx citas relativas, de alguna manera a "lo espacial". 
consideración de determinismos naturales y culturales en general y una adecuación especial a las formas particulares en "que el determinismo real en su conjunto está estructurado en cada cáso.

Por último, digamos que un enfoque de in restigación opuesto a las concepciones apologéticas aún imperantes en el campo implicará no sólo mostrar cómo la realidad es efectivamente, no sólo mostrar por qué es, cómo es y no de otra manera, sino ambos al mismo tiempo y también mostrar lo que podría ser, no a partir de un deseo idealista, sino del análisis de los contradictorios procesos actuales. 